\title{
GMR
}

\section{A retrospective analysis of surgical treatment of mesh infection after repair of ventral hernia or defect}

\author{
F.-D. Liu, J.-Y. Li, S. Yao and Y. Zhang \\ Department of General Surgery, General Hospital of Chinese PLA, \\ Medical School of Chinese PLA, Beijing, China \\ Corresponding author: F.-D. Liu \\ E-mail:wentiancn@163.com \\ Genet. Mol. Res. 14 (4): 14387-14395 (2015) \\ Received July 4, 2015 \\ Accepted October 2, 2015 \\ Published November 18, 2015 \\ DOI http://dx.doi.org/10.4238/2015.November.18.2
}

ABSTRACT. This study aimed to summarize our experience in surgical treatment of mesh infection after repair of ventral hernia or defect. A retrospective analysis was conducted on clinical data of 22 patients who accepted surgical treatment of mesh infection after ventral hernia or defect repair. Included were 16 cases of infection after incisional hernia repair, 5 cases of infection after abdominal wall defect repair following abdominal wall tumor resection, and 1 case of infection with fistula caused by a parastomal hernia of an ileal neobladder repair with a prosthetic patch. All patients had received local dressing treatment for 2 to 24 months but were not healed. The affected mesh was removed successfully in all patients. Six patients had abdominal wall repair using the component separation technique; 4 patients were treated by strengthened repair with polypropylene mesh; 10 patients were repaired with human acellular dermal matrix; 1 patient received local dressing changes and vacuum sealing drain treatment without repair; and 1 patient received wound closure without strengthened repair. The postoperative hospital stay was 9-29 days (mean 16 days). After treatment, 19 patients recovered with primary wound healing and 3 patients recovered with secondary healing. All patients were 
followed up for 6-38 months (mean 26 months), and no ventral hernia or defect recurred except 1 case of lower abdominal bulge. Mesh infections after ventral hernia or defect repair are difficult to treat using prosthetic materials. For satisfactory results, surgery should be performed according to the specific condition of the individual.

Key words: Mesh infection; Surgical treatment; Hernia; Defect repair

\section{INTRODUCTION}

With the increasing popularity of tension-free hernioplasty, several types of prosthetic mesh have been applied widely in clinical practice. Clearly, the development and application of prosthetic mesh has decreased the ventral hernia relapse rate, and improvements in prosthetic mesh have accelerated progress in hernia repair and even abdominal wall surgery. However, we must pay attention to the complications associated with prosthetic meshes, especially that of mesh infection. Once mesh infection occurs, it is very difficult to cure (Stremitzer et al., 2010; Sanchez et al., 2011; Akyol et al., 2013); the situation usually requires complete removal of the mesh and sometimes multiple surgical treatments. Furthermore, after infected mesh removal, the problem of abdominal wall reconstruction still exists. To study this, we analyzed the clinical data of 22 patients with mesh infection who underwent surgical treatment in our department between June 2008 and September 2012.

\section{MATERIAL AND METHODS}

\section{Clinical data}

The study group consisted of 22 patients: 13 males and 9 females, aged 22 to 78 years (mean 49.1 years). Included were 16 cases of mesh infection after incisional hernia repair (Figures 1-3), 5 cases of abdominal wall defects caused by abdominal wall tumor resection, and 1 mesh infection and urinary fistula caused by a parastomal hernia of an ileal neobladder repair with a prosthetic patch.

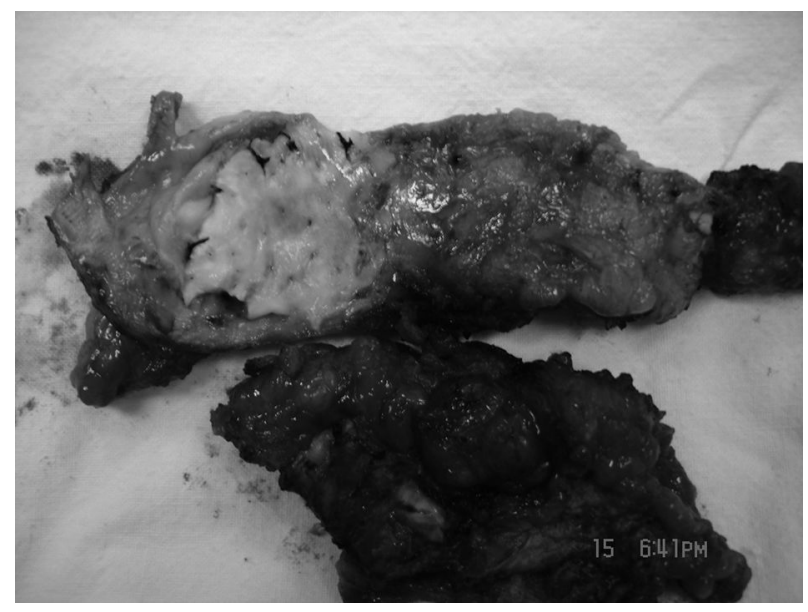

Figure 1. Mesh infection and intestinal erosion after incisional hernia repair. 


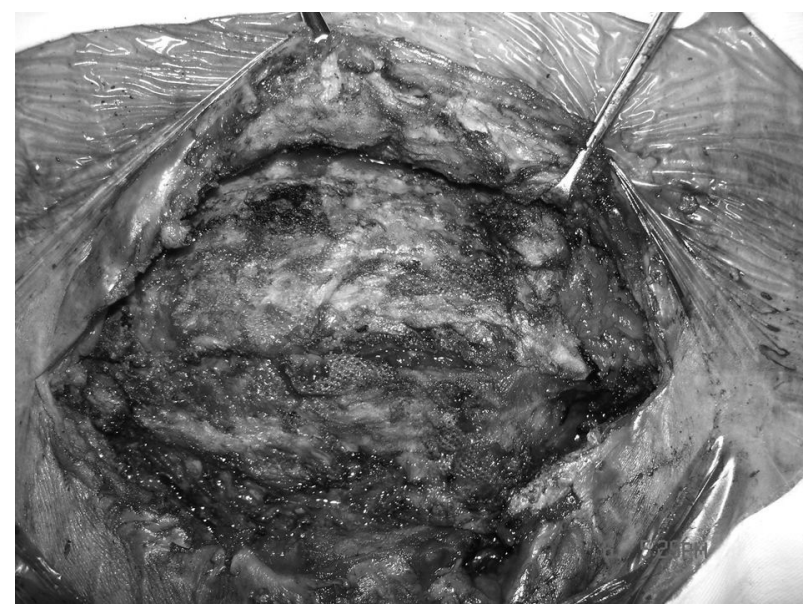

Figure 2. Chronic abscess after incisional hernia repair with prosthetic mesh.

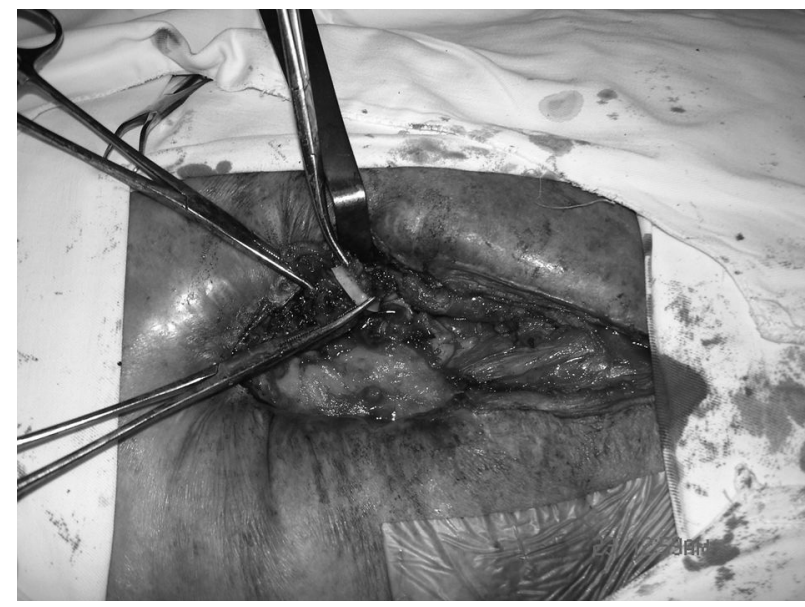

Figure 3. Mesh infection and exposure after incisional hernia repair.

All patients were treated with local dressing changes by the primary operative surgeon for 3 to 14 months, but their wounds did not heal. These patients accepted radical removal of the infected mesh and abdominal wall reconstruction in our department. This study was conducted in accordance with the Declaration of Helsinki and was approved by the Ethics Committee of the First Affiliated Hospital, General Hospital of Chinese PLA. Written informed consent was obtained from all participants.

\section{Surgical methods}

All patients underwent general anesthesia. Highlights of the surgical approach are as follows: The infected mesh was removed as completely as possible, and infected and necrotic tissue was cleared out. The wound surface of the infection site was washed multiple times with 
saline and metronidazole solution. After mesh removal, one of the following treatment strategies was chosen, according to level of the abdominal wall defect: component separation technique, abdominal wall repair with polypropylene mesh, abdominal wall repair using human acellular dermal matrix, dressing change and vacuum sealing drain for the infected wound surface without reconstruction. A latex drainage tube was placed within the surgical site for all wounds or repaired meshes.

\section{Postoperative handling}

Standard broad-spectrum antibiotics were administered during the operation and changes at the incision site were closely monitored postoperatively. On postoperative days 3-5, bedside ultrasound examination was performed. For any indication of effusion at the site, local puncture suction was performed, and the patient was reexamined as needed. The drainage tube was removed when the drainage fluid level dropped below $20 \mathrm{~mL}$ per day. Patients without repair were treated with a vacuum sealing drain at the incision site and daily dressing changes.

\section{RESULTS}

In all patients, the infected mesh was completely removed. Six patients were treated with the component separation technique; 4 patients were repaired by reinforcement with polypropylene mesh; 10 patients were repaired with acellular dermal matrix mesh (Figure 4); 1 patient was not repaired and was treated with a vacuum sealing drain at the incision site and local dressing changes (Figure 5); and 1 patient had mesh removal followed by incision closure and suturing without repair (Figure 6).

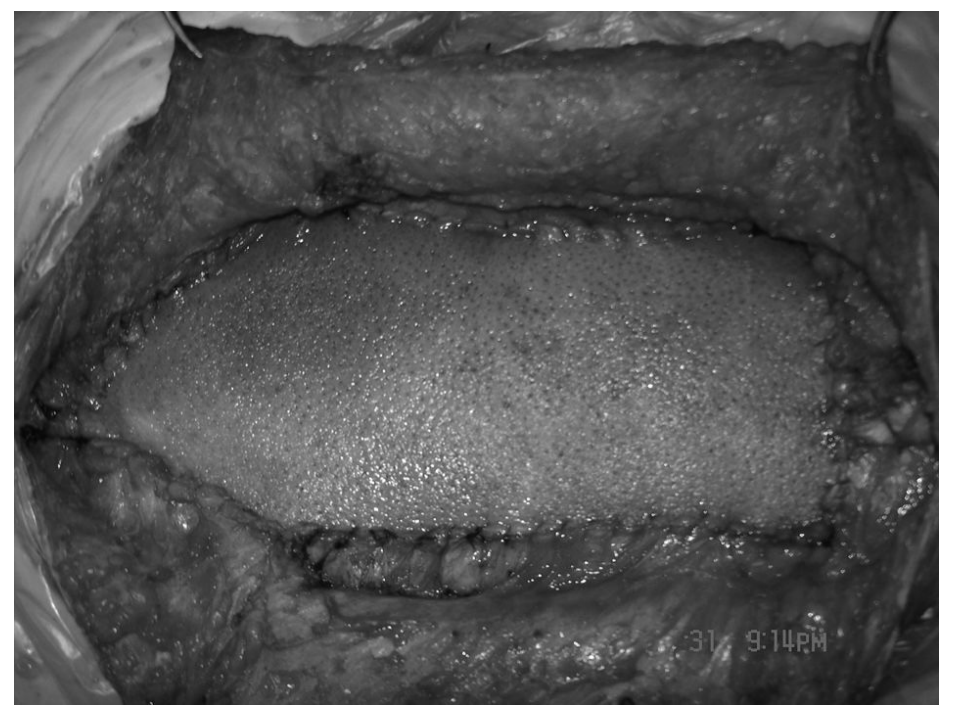

Figure 4. Abdominal wall repair using human acellular dermal matrix after infected mesh resection. 


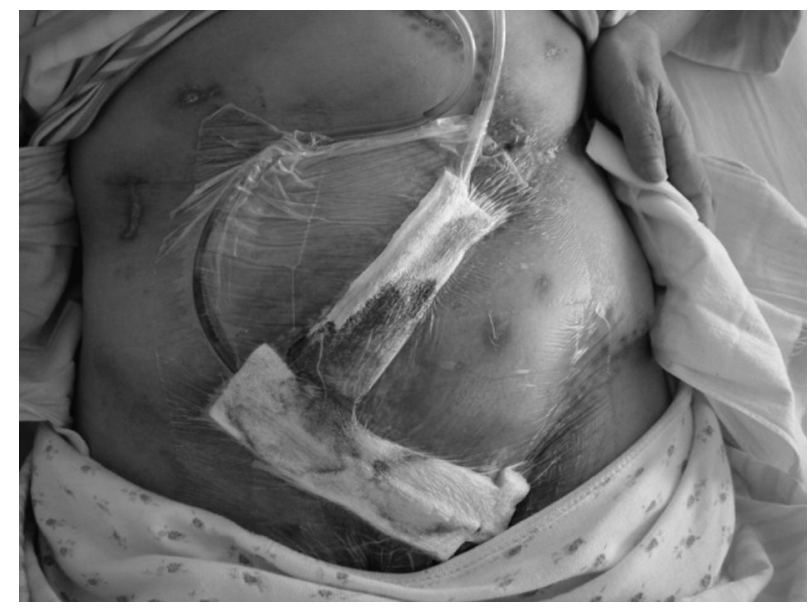

Figure 5. Vacuum sealing drain at the incision site, and dressing change of the infected wound surface, after infected mesh removal without abdominal wall reconstruction.

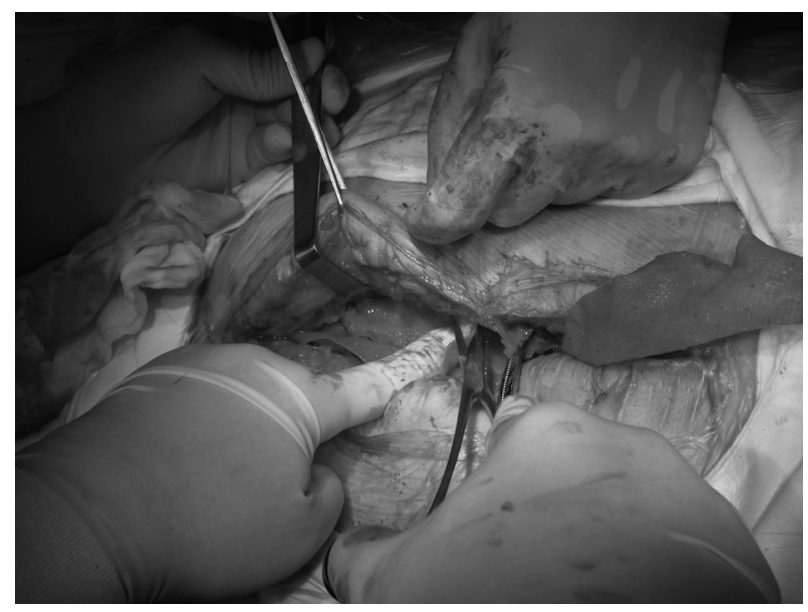

Figure 6. Closure of incision after infected mesh removal without repair.

The postoperative hospital stay was 9-25 days with a mean of 14 days. Nineteen patients achieved primary wound healing, and 3 patients achieved secondary healing after local dressing changes. Patients were followed up for 6-38 months with a mean of 23 months. One patient with a history of resection of a giant fibroma had mesh infection removal without repair, because of fibroma recurrence in the abdomen and mediastinum, and developed a slight lower abdominal bulge. The other patients had no ventral hernia relapse.

\section{DISCUSSION}

Currently, several types of prosthetic mesh are widely used for repairing ventral hernias or defects, such as incisional hernia, parastomal hernia, umbilical hernia, inguinal hernia, and 
abdominal wall weakness and defect due to abdominal tumor resection. Compared with traditional methods, prosthetic meshes have the advantages of decreased trauma and postoperative relapse rates (Luijendijk et al., 2000). However, despite the lower relapse rate with prosthetic meshes, mesh-related complications, such as mesh infection, intestinal adhesion, intestinal obstruction, and enterocutaneous fistula present new problems (Brown et al., 2013; Sahoo et al., 2013). How to deal with these problems is important for us to address.

Globally, one million prosthetic meshes are implanted every year (Junge et al., 2005). The postoperative infection rate in open inguinal hernia mesh repair has been reported as high as $9 \%$ (Yerdel et al., 2001), whereas laparoscopic ventral hernia mesh repair has a postoperative mesh infection rate of $0.7-2 \%$, and open ventral hernia mesh repair has an infection rate as high as $7-18 \%$ (Ríos et al., 2001; Itani et al., 2010). Mesh infection is a great challenge for surgeons. It is well known that the fundamental principle for approaching this problem is to remove the mesh completely. However, the goals of surgery are not only to remove the infected mesh, control infection, and achieve wound healing, but also to address the abdominal wall defect and weakness to prevent hernia relapse and eventration at the surgical site. Handling of this problem has been controversial, and surgeons have tried different approaches, including primary removal of the infected mesh without abdominal wall repair and performance of a second surgery if hernia relapse or eventration occurred; or primary removal of the infected mesh and repair of the weak areas using autologous tissue or biosynthetic meshes. Until now, no satisfactory standard treatment has existed.

To treat a mesh infection, the first task is to identify the type of mesh, method of implantation, and method of repair. If the mesh was placed with onlay technique (mesh before muscular fasciae), the infection would be shallow and would not involve the abdominal cavity; thus, local surgical treatment such as complete removal of the mesh would achieve healing, and the surgery would not enter into the abdominal cavity, thereby avoiding impact on the intestines and increased chance of hernia relapse.

If sublay (mesh between muscular fasciae and peritoneum) or IPOM technique (intraperitoneal onlay mesh) were used, surgical removal of the mesh might damage the intestines and possibly lead to postoperative hernia or eventration. In fact, many patients who have developed mesh infections after these interventions have undergone long-term external wound dressing change treatment without recovery, and the scars around the abdominal wall incision undergo significant fibrillation and hardening. After mesh removal, it becomes almost impossible to separate tissues around the incision site, and the intestines underneath are exposed. The intestinal surface fibrillated without a clear border, and adhesions cannot be separated without damaging the intestines. One patient in our group was exactly as described - no repair was performed after mesh removal for the reasons stated above, and the incision achieved secondary healing after external medication treatment. No hernia or eventration was observed, possibly because the abdominal wall around the incision was hardened as a result of long-term hyperplasia. Follow-up was 39 months, and the local abdominal wall slightly softened around the incision site without hernia or eventration. Whether a hernia will eventually occur after the local scar softens further is still pending follow-up.

The use of autologous tissue, after dissociation, for reinforced repair is another viable approach to surgery for mesh infection. After dissociation, a rectus abdominis flap could close a large midline defect, making it potentially part of a successful surgical intervention after mesh removal. According to existing literature, when using this method, also called component separation method, the hernia relapse rate is low (Saulis and Dumanian, 2002; Kim and Kim, 2011; Krpata 
et al., 2012; Espinosa-de-los-Monteros et al., 2013; Morris and LeBlanc, 2013; Wang and Singh, 2013). Even with the low relapse rate, this method is not appropriate for all types of repair after mesh removal (e.g., if the defect is too large or serious scar hyperplasia has made dissociation nearly impossible). In our group, the component separation technique was applied in 6 cases, and all incisions achieved primary healing postoperatively. Furthermore, at follow-ups, no hernia relapses occurred. Mathes et al. (2000) reported on 100 cases in which the tensor fasciae latae was used to reconstruct abdominal wall damage after infected mesh removal. In these cases, using the component separation technique had its own limitations: the tensor fasciae latae was difficult to dissociate for umbilical defect repair; and after the flap was overturned, the dissociated vessel pedicle at the inguinal area exerted pressure on the femoral vein, increasing the risk of deep vein thrombosis.

A repeat repair with prosthetic mesh is another option following mesh infection, but placing a piece of prosthetic mesh at a highly contaminated site or on an infected wound surface is highly risky, and according to previous literature, the infection rate with this approach could be as high as $50-90 \%$ (Dayton et al., 1986). Polypropylene mesh has higher anti-infective properties compared with types of prosthetic meshes currently in use, but its placement requires a surgeon highly experienced in hernia and abdominal wall repair. In our group, this method was used in 4 patients, and all incisions achieved primary healing postoperatively. Polypropylene mesh is only appropriate for patients with a relatively complete peritoneum because it cannot have direct contact with visceral organs.

Acellular dermal matrix is a new repair material that emerged from developments in immunology and tissue engineering. The source of this material is dermis from human cadavers, processed to eliminate all cellular components in the dermal layer while retaining the extracellular matrix and basement membrane. The extracellular matrix frame formation promotes growth of fibroblasts and collagen deposits and maturation, and these changes occur when the material is implanted in the human body (Sclafani et al., 2002; An et al., 2004; Silverman et al., 2004). Complete preservation of the extracellular matrix and progressive blood supply reconstruction allow the material to keep its own structure and differentiate into autologous tissue at the same time. An animal model has proven that early blood supply reconstruction increases the anti-infective properties of the implanted material (Menon et al., 2003). Therefore, the most important advantage of acellular dermal matrix is a high tolerance of infection around the site without a decrease in tensile strength. Additionally, it will not lead to permanent foreign object residual at the repair site. The mechanical properties of this material are also promising and include anti-tensile strength, elasticity, and flexibility (Janis and Nahabedian, 2012). On the basis of these properties, acellular dermal matrix is an ideal material for ventral hernia repair and may substitute for prosthetic meshes under certain conditions. In our group, acellular dermal matrix was used to repair abdominal wall defects or hernias in 10 patients. All achieved primary healing except 1 patient who had a postoperative infection of a parastomal hernia repaired by polypropylene mesh, complicated by a urinary fistula. We surgically removed the infected mesh and cystectomy ileum, reconstructed the ileal bladder stoma, and used acellular dermal matrix for primary repair. Postoperatively, a sinus partially formed at the incision site, and secondary healing was achieved after dressing changes. On follow-up, no hernia relapse was reported. The disadvantage of acellular dermal matrix is the cost - it is very expensive, and large defect repairs might require several pieces, making it unrealistic for most patients.

The issues of mesh infection after ventral hernia repair and chronic mesh exposure are 
not emphasized enough. Many patients encounter these problems, and despite wound dressing treatment by the primary surgeon, their infections are still not controlled. These patients then seek further treatment at other hospitals; therefore, the incidence of mesh infection may be higher than is reported. In our study, we described some of our experience with this issue. We believe that treatment for mesh infection, such as with autologous tissue repair, prosthetic mesh application, or acellular dermal matrix implantation, should be individualized according to the patient's condition. However, none of these treatment methods are perfect; therefore, preventing mesh infection is of primary importance.

\section{ACKNOWLEDGMENTS}

This study was performed without any pharmaceutical or industry support. The authors declare that they have no conflicts of interest.

\section{REFERENCES}

Akyol C, Kocaay F, Orozakunov E, Genc V, et al. (2013). Outcome of the patients with chronic mesh infection following open inguinal hernia repair. J. Korean Surg. Soc. 84: 287-291.

An G, Walter RJ and Nagy K (2004). Closure of abdominal wall defects using acellular dermal matrix. J. Trauma 56: 1266-1275.

Brown RH, Subramanian A, Hwang CS, Chang S, et al. (2013). Comparison of infectious complications with synthetic mesh in ventral hernia repair. Am. J. Surg. 205: 182-187.

Dayton MT, Buchele BA, Shirazi SS and Hunt LB (1986). Use of an absorbable mesh to repair contaminated abdominal-wall defects. Arch. Surg. 121: 954-960.

Espinosa-de-los-Monteros A, Domínguez I, Zamora-Valdés D, Castillo T, et al. (2013). Closure of midline contaminated and recurrent incisional hernias with components separation technique reinforced with plication of the rectus muscles. Hernia 17: 75-79.

Itani KM, Hur K, Kim LT, Anthony T, et al. (2010). Comparison of laparoscopic and open repair with mesh for the treatment of ventral incisional hernia: a randomized trial. Arch. Surg. 145: 322-328.

Janis JE and Nahabedian MY (2012). Acellular dermal matrices in surgery. Plast. Reconstr. Surg. 130: 7S-8S.

Junge K, Rosch R, Klinge U, Krones C, et al. (2005). Gentamicin supplementation of polyvinylidenfluoride mesh materials for infection prophylaxis. Biomaterials 26: 787-793.

Kim Z and Kim YJ (2011). Components separation technique for large abdominal wall defect. J. Korean Surg. Soc. 80: S63-S66.

Krpata DM, Blatnik JA, Novitsky YW and Rosen MJ (2012). Posterior and open anterior components separations: a comparative analysis. Am. J. Surg. 203: 318-322.

Luijendijk RW, Hop WC, van den Tol MP, de Lange DC, et al. (2000). A comparison of suture repair with mesh repair for incisional hernia. N. Engl. J. Med. 343: 392-398.

Mathes SJ, Steinwald PM, Foster RD, Hoffman WY, et al. (2000). Complex abdominal wall reconstruction: a comparison of flap and mesh closure. Ann. Surg. 232: 586-596.

Menon NG, Rodriguez ED, Byrnes CK, Girotto JA, et al. (2003). Revascularization of human acellular dermis in full-thickness abdominal wall reconstruction in the rabbit model. Ann. Plast. Surg. 50: 523-527.

Morris LM and LeBlanc KA (2013). Components separation technique utilizing an intraperitoneal biologic and an onlay lightweight polypropylene mesh: "a sandwich technique". Hernia 17: 45-51.

Ríos A, Rodríguez JM, Munitiz V, Alcaraz P, et al. (2001). Antibiotic prophylaxis in incisional hernia repair using a prosthesis. Hernia 5: 148-152.

Sahoo MR, Bisoi S and Mathapati S (2013). Polypropelene mesh eroding transverse colon following laparoscopic ventral hernia repair. J. Minim. Access Surg. 9: 40-41.

Sanchez VM, Abi-Haidar YE and Itani KM (2011). Mesh infection in ventral incisional hernia repair: incidence, contributing factors, and treatment. Surg. Infect. 12: 205-210.

Saulis AS and Dumanian GA (2002). Periumbilical rectus abdominis perforator preservation significantly reduces superficial wound complications in "separation of parts" hernia repairs. Plast. Reconstr. Surg. 109: 2275-2280.

Sclafani AP, McCormick SA and Cocker R (2002). Biophysical and microscopic analysis of homologous dermal and fascial materials for facial aesthetic and reconstructive uses. Arch. Facial. Plast. Surg. 4: 164-171. 
Silverman RP, Li EN, Holton LH 3rd, Sawan KT, et al. (2004). Ventral hernia repair using allogenic acellular dermal matrix in a swine model. Hernia 8: 336-342.

Stremitzer S, Bachleitner-Hofmann T, Gradl B, Gruenbeck M, et al. (2010). Mesh graft infection following abdominal hernia repair: risk factor evaluation and strategies of mesh graft preservation. A retrospective analysis of 476 operations. World J. Surg. 34: 1702-1709.

Wang HD and Singh DP (2013). The use of indocyanine green angiography to prevent wound complications in ventral hernia repair with open components separation technique. Hernia 17: 397-402.

Yerdel MA, Akin EB, Dolalan S, Turkcapar AG, et al. (2001). Effect of single-dose prophylactic ampicillin and sulbactam on wound infection after tension-free inguinal hernia repair with polypropylene mesh: the randomized, double-blind, prospective trial. Ann. Surg. 233: 26-33. 